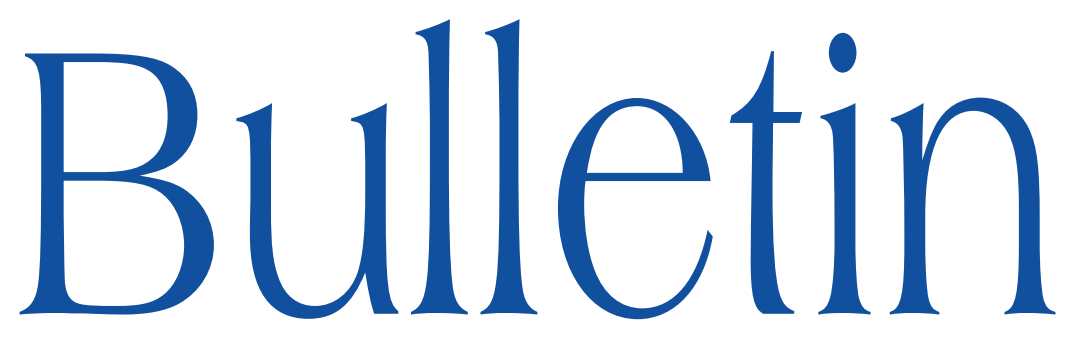

de la SOCIÉTÉ MATHÉMATIQUE DE FRANCE

\title{
ESTIMATIONS \\ DE LA FONCTION MAXIMALE DE HARDY-LITTLEWOOD
}

Noël Lohoué

\section{Tome 135 Fascicule 3}




\title{
ESTIMATIONS DE LA FONCTION MAXIMALE DE HARDY-LITTLEWOOD
}

\author{
PAR NOËL LOHOUÉ
}

\begin{abstract}
RÉsumÉ. - On montre que la fonction maximale de Hardy-Littlewood est de type $(p, p)$ sur certains groupes de Lie et variétés de Cartan-Hadamard.

Abstract (Estimations of the maximal Hardy-Littlewoord function)

We prove $L^{p}$ boundness of Hardy-Littlewood maximal functions on a class of Lie groups and Cartan-Hadamard manifolds.
\end{abstract}

\section{Introduction}

On se donne un espace métrique $M$ muni d'une distance $\delta$ et d'une mesure $\mathrm{d} \sigma$ qui charge les boules de $M$ de centre arbitraire et de rayon quelconque d'une masse finie. Si $f: M \rightarrow \mathbb{C}$ est une fonction $\mathrm{d} \sigma$ mesurable, on s'intéresse à la fonction $f^{*}$ définie par

$$
f^{*}(x)=\sup _{r>0} \frac{1}{\left|B_{x}(r)\right|} \int_{B_{x}(r)}|f(y)| \mathrm{d} \sigma(y)
$$

où $B_{x}(r)$ désigne la boule, au sens de $\delta$, de centre $x$ et de rayon $r,\left|B_{x}(r)\right|$ sa mesure.

Texte reçu le 25 octobre 2005, révisé le 19 décembre 2006

NoËL Lohoué, Université de Paris-Sud, Mathématique, bât. 425, UMR 8628 du CNRS, 91405 Orsay Cedex (France) • E-mail : noel.lohoue@math.u-psud.fr Classification mathématique par sujets (2000). — 22E80; 43A90, 60B90. Mots clefs. - Fonction maximale. 
On voudrait prouver des estimations du style, pour $1<p<\infty$, il existe une constante $C(p)$ telle que

$$
\left\|f^{*}\right\|_{p} \leq C(p)\|f\|_{p}
$$

Ceci est un vieux thème et l'on sait bien que cette inégalité ne peut pas être prouvée en toute généralité pour tout triplet $(M, \delta, \mathrm{d} \sigma)$.

On s'intéresse dans cet article à deux situations particulières où l'on veut utiliser la géométrie de $(M, \delta, \mathrm{d} \sigma)$ pour donner une réponse positive à la question ci-dessous.

a) $M$ est un groupe de Lie non moyennable, la distance $\delta$ est la distance de contrôle associée à un système de champs de Hörmander invariants à gauche et $\mathrm{d} \sigma$ est la mesure de Haar sur $G$, bi-invariante.

b) $M$ est une variété de Cartan Hadamard avec quelques contraintes sur la courbure; la distance associée sur $M$ est la distance riemannienne et la mesure est induite par cette structure.

Ces deux exemples ont on point commun; le volume des boules de grand rayon croît exponentiellement, ce qui rend toutes les techniques usuelles inutilisables : on ne peut faire appel au lemme de recouvrement que pour les boules de rayon plus petit qu'un nombre donné. Par contre, si $M$ est à courbure de Ricci positive ou nulle, on sait qu'alors $M$ est un espace de nature homogène et on a droit à tout.

L'énoncé général suivant indique la direction que l'on veut suivre.

Proposition 1. - Soit $(M ; \delta)$ un espace métrique muni d'une mesure $\mathrm{d} \sigma$ comme décrit ci-dessus. On suppose qu'il existe une constante $0<C<\infty$ telle que pour tout couple de point $(x, y)$,

$$
C^{-1}<\frac{\left|B_{x}(r)\right|}{\left|B_{y}(r)\right|}<C \quad \text { et } \quad\left|B_{x}(2 r)\right|<C\left|B_{x}(r)\right|,
$$

pour $0<r<1$. Soit 0 un point distingué de $M$. Alors, pour tout $\varepsilon>0$, il existe pour tout $1<p<\infty$, une constante $C_{\varepsilon}(p)$ telle que

$$
\left\|\left(1+\left|B_{0}(\widetilde{\delta})\right|\right)^{-\varepsilon} f^{*}\right\|_{p} \leq C_{\varepsilon}(p)\|f\|_{p} .
$$

ò̀ $\widetilde{\delta}$ est la fonction $\widetilde{\delta}(x)=\delta(x, 0)$.

Les deux résultats sur lesquels on veut s'attarder sont indiqués ci-dessous.

REMARQUE. - La proposition 1 s'applique si $G$ est un groupe unimodulaire, $\delta$ une métrique invariante à gauche sur $G$ et $\mathrm{d} \sigma$ la mesure de Haar. Dans le premier résultat on va essayer d'enlever $\varepsilon$ pour un cas particulier. Pour énoncer ce premier résultat que l'on veut démontrer on aura besoin de quelques notations. 
Notations. - Soit $G$ un groupe de Lie connexe, unimodulaire, non compact, non moyennable. On note

$$
G=H \odot G_{0}
$$

sa décomposition de Levi. On suppose que la partie résoluble distinguée $H$ de $G$ est unimodulaire, à croissance polynomiale, de dimension $D$ à l'infini (voir [9] pour la notion de dimension à l'infini). On suppose aussi que la partie semi-simple $G_{0}$ est de centre fini, connexe, de dimension topologique $m_{0}$.

On note $\mathcal{G}$ l'algèbre de Lie de $G, \mathcal{H}$ celle de $H, \mathcal{G}_{0}$ celle de $G_{0}$ et $\sigma$ la représentation de $\mathcal{G}_{0}$ sur $\mathcal{H}$. Alors $\mathcal{H}=\bigoplus_{i=1}^{m} \mathcal{H}_{i}$ où $\sigma_{\mid \mathcal{H}_{i}}=\sigma_{i}$ est une sousreprésentation irréductible de $\mathcal{G}_{0}$ et de poids $\alpha_{i_{1}}, \ldots, \alpha_{m_{i, 1}}, m_{i}$. On pose

$$
\Theta=\sum_{\substack{i=1 \\ j=1}}^{m, m_{i}} m_{i j} \alpha_{i j}
$$

Par la suite on va considérer une distance de contrôle associée à un système de Hörmander particulier pour des simples raisons d'exposition. La considération d'un système général alourdirait considérablement le texte sans simplifier la compréhension des idées développées.

Notons $\mathcal{G}_{0}=k_{0} \oplus p_{0}$ une décomposition de Cartan de $\mathcal{G}_{0}, K_{0}$ le sous-groupe compact maximal associé à $k_{0}$. On considère sur $p_{0}$ une base orthonormée pour le produit scalaire $\langle X, Y\rangle_{0}=-B\left(X, \Theta_{0} Y\right)$ où $B$ est la forme de Killing, $\Theta_{0}$ l'involution de Cartan.

Soit $\widetilde{X}_{1}, \ldots, \widetilde{X}_{k}$ un système de Hörmander de champs invariants à gauche sur $G$ tels que :

- $X_{1}, \ldots, X_{\ell}$ sont dans $\mathcal{H}$ et $\widetilde{X}_{1}, \ldots, \widetilde{X}_{\ell}$ est un système de Hörmander sur $H$;

- $X_{\ell+1}, \ldots, X_{s}$ sont dans $k_{0}$ et $X_{s+1}, \ldots, X_{k}$ dans $p_{0}$ et les $X_{1}, \ldots, X_{k}$ sont linéairement indépendants.

On considère sur $p_{0}$ le produit scalaire $\langle., .\rangle_{1}$ tel que $\left\langle X_{i}, X_{j}\right\rangle_{1}=\delta_{i j}$ pour $s+1 \leq i, j \leq k$. On prolonge ce produit scalaire à $\mathcal{G}$ de telle sorte que $p_{0}$ et $k_{0}$ soient orthogonaux.

On pose

$$
\gamma_{0}=\sup _{k^{\prime} \in K_{0}}\left\|\operatorname{Ad}\left(k^{\prime}\right)\right\|
$$

où $\left\|\operatorname{Ad}\left(k^{\prime}\right)\right\|$ est la norme de $\operatorname{Ad}\left(k^{\prime}\right)$ agissant sur $p_{0}$ muni du produit scalaire $\langle., .\rangle_{1}$.

Soient

$$
\|\alpha\|=\sup _{i, j}\left\|\alpha_{i j}\right\|, \quad \beta=\gamma_{0}^{2}\|\alpha\| .
$$

On note $\delta_{0}$ la distance sur $G_{0}$, invariante à gauche dont la restriction au plan tangent à l'origine correspond au produit scalaire $\langle X, Y\rangle_{0}$. 
Soient $\mathcal{A}^{+}$une chambre de Weyl positive associée à la décomposition de $\mathcal{G}_{0}=$ $k_{0} \oplus p_{0}$ et $2 \rho$ la somme des racines positives comptées avec leurs multiplicités relatives à $\mathcal{A}^{+}$.

Soit $\mathcal{H}_{i j}^{1}$ l'espace radiciel de poids $\alpha_{i j} \geq 0$ sur $\mathcal{A}$, si $\mathcal{H}_{i j}^{2}$ est l'espace radiciel de poids $-\alpha_{i j}$; on suppose que $K$ est transitif sur les sphères de $\mathcal{H}_{i j}^{1} \oplus \mathcal{H}_{i j}^{2}$.

Sous ces conditons on a l'énoncé suivant :

THÉORÈme 2. - On suppose qu'il existe $0<\varepsilon<\frac{1}{2}$ tel que

$$
0<\frac{D \beta}{\|2 \rho+\Theta\|_{0}-2\|\rho\|_{0}(1-\varepsilon)}<1 .
$$

Alors pour tout $p>1 /(1-\varepsilon)$ il existe $C(p)$ telle

$$
\left\|f^{*}\right\|_{p} \leq C(p)\|f\|_{p}
$$

où $\|2 \rho+\Theta\|_{0}, 2\|\rho\|_{0}$ est la norme de $2 \rho+\Theta, 2 \rho$ donnée par le produit scalaire $\langle., .\rangle_{0}$.

REMARque. - L'exemple type où l'hypothèse du théorème s'applique est $\mathbb{R}^{2}$ (s) $\mathrm{SL}(2, \mathbb{R})$. Les groupes concernés sont non moyennables et non semisimples. Ils échappent à un contrôle brutal par Kunze-Stein. On va utiliser de la géométrie pour s'y ramener.

$\mathrm{Au}$ cours de la preuve du théorème on montrera aussi l'énoncé suivant :

Proposition 3. - Soit $\delta$ une métrique comme ci-dessus. Soit $\chi_{r}$ la fonction caractéristique de la boule de rayon $r$, notée $B_{r}$; on considère $\beta_{r}=\chi_{r} /\left|B_{r}\right|$ où $\left|B_{r}\right|$ est le volume de $B_{r}$. Si $1 /(1-\varepsilon)<p<1 / \varepsilon$, il existe $\alpha(p)>0$ telle que

$$
\left\|\beta_{r}\right\|_{p \rightarrow p} \leq C_{1} \mathrm{e}^{-\alpha(p) r} \quad \text { où } C_{1} \text { est une constante }
$$

La proposition 3 est un cas particulier d'une conjecture de [8].

ThÉorème 4. - $M$ est une variété de Cartan-Hadamard et les $L^{p}$ que l'on considère sont relatifs à la mesure riemannienne. On fait sur $M$ les hypothèses suivantes :

a) La courbure de Ricci de $M$ est minorée par $-b^{2}$ où $b \in \mathbb{R}^{*}$.

b) Pour tout $x$ fixé, on note $\Theta_{x}(s, \omega)$ la densité de volume en coordonnées polaires exponentielles au point $x$ de $M$ et $H_{x}(r, \omega)$ la courbure moyenne de la sphère de centre $x$ et de rayon $r$ évaluée au point $\exp _{x} r \omega$, et l'on suppose que

$$
\sigma_{0}=\inf _{x, r, \omega} H_{x}(r, \omega)>0
$$


c) Soit $\psi_{x}(y)=\mathrm{e}^{-\phi_{x}(\delta(x, y))}=\left[\int_{\Sigma^{n-1}} \Theta_{x}(r, \omega) \mathrm{d} \omega\right]^{-1}$ avec $y=\exp _{x} r \omega$, l'inverse de l'aire de la sphère de centre $x$ et de rayon $r$ pour la mesure induite. On suppose qu'il existe $0<\sigma<\sigma_{0}$ telle que

$$
-\frac{\mathrm{d}^{2} \varphi_{x}(r)}{\mathrm{d} r^{2}}+\left(\frac{\mathrm{d} \varphi_{x}(r)}{\mathrm{d} r}\right)^{2}-\tau \frac{\mathrm{d} \varphi_{x}}{\mathrm{~d} r}+\sigma^{2} \geq 0
$$

ò̀ $\tau=\sup _{\|\omega\|=1} H_{x}(r, \omega)$ et que

$$
\lim _{x \rightarrow \infty} \frac{\psi(x ; y)}{G_{\sigma(x, y)}}<C_{1}
$$

où $G_{\sigma}(x, y)$ est la fonction de Green de $\Delta+\sigma^{2}$ et $C_{1}$ une constante qui ne dépend pas de $y$.

Sous les hypothèses a), b), c), il existe une constante $1<p_{0}<\infty$ telle que pour tout $p_{0}<p$ il existe $C(p)$ telle que

$$
\left\|f^{*}\right\|_{p} \leq C(p)\|f\|_{p} .
$$

Corollaire 5. - On suppose que la courbure $C$ de $M$ vérifie l'inégalité

$$
-b^{2} \leq C \leq-a^{2}, \quad \text { avec } \quad 0<a<b<\frac{5 a}{4} .
$$

Si $p>p_{0}=a /(3 a-2 b)$ alors il existe $C(p)$ tel que $\left\|f^{*}\right\|_{p} \leq C(p)\|f\|_{p}$.

Ce corollaire est établi dans [4].

\section{Démonstration des résultats}

L'idée générale de la preuve consiste à utiliser la troncature de [2] :

$$
\begin{aligned}
f^{*}(x) \leq \sup _{r \leq 1} & \frac{1}{\left|B_{x}(r)\right|} \int_{B_{x}(r)}|f(y)| \mathrm{d} \sigma(y) \\
& +\sup _{r \geq 1} \frac{1}{\left|B_{x}(r)\right|} \int_{B_{x}(r)}|f(y)| \mathrm{d} \sigma(y)=f_{1}^{*}(x)+f_{2}^{*}(x) .
\end{aligned}
$$

Sous les hypothèses de la proposition 1 d'après les résultats classiques pour tout $1<p<\infty$, il existe une constante $C(p)$ telle que

$$
\left\|f_{1}^{*}\right\|_{p} \leq C(p)\|f\|_{p} .
$$

Cette inégalité vaut pour les boules du théorème 2 d'après [7] ainsi que pour celles du théorème 4 car $M$ est à géométrie bornée et l'on utilise un lemme de recouvrement pour les boules de rayon plus petit que 1 . 
Dans les trois cas, il faut s'occuper de $f_{2}^{*}(x)$. On remarque dans les trois cas que

$$
f_{2}^{*}(x) \leq \int_{M} \inf \left\{\left|B_{x}(1)\right|^{-1}, \frac{1}{\left|B_{x}(\delta(x, y))\right|}\right\}|f(y)| \mathrm{d} \sigma(y) .
$$

Mais par hypothèse $\left|B_{x}(1)\right| \geq C^{-1}\left|B_{0}(1)\right|$ où 0 est un point distingué sur $M$, comme $\left|B_{x}(r)\right|$ est croissante $\mid B_{x}\left(\delta(x, y)\left|\geq C^{-1}\right| B_{0}(1) \mid\right.$ pour $\delta(x, y) \geq 2$ et

$$
\begin{aligned}
f_{2}^{*}(x) \leq C_{1} & \int_{1<\delta(x, y) \leq 2}|f(y)| \mathrm{d} \sigma(y) \\
& +C_{2} \int_{\delta(x, y) \geq 2} \frac{1}{\mid B_{x}(\delta(x, y))}|f(y)| \mathrm{d} \sigma(y)=f_{3}^{*}(x)+f_{4}^{*}(x) .
\end{aligned}
$$

Comme $\left\|f_{3}^{*}\right\|_{p} \leq C_{p}\|f\|_{p}$ pour tout $1 \leq p \leq \infty$, il faut étudier $f_{4}^{*}$, que l'on note $S(f)$ pour simplifier.

2.1. Preuve de la la proposition 1 . - On considère le noyau

$$
K(x, y)=\frac{1}{\left|B_{x}(\delta(x, y))\right|} \mathbf{1}_{\delta(x, y) \geq 2}
$$

où $\mathbf{1}_{\delta(x, y) \geq 2}$ est la fonction caractéristique de $\{x, y \in M ; \delta(x, y) \geq 2\}$.

Alors pour $x$ fixé (resp. $y$ fixé), $K(x, y)$ comme fonction de $y$ (resp. fonction de $x$ ) est dans $L^{1, \infty}(M)$ uniformément en $x$ (resp. en $y$ ). On a en effet

$$
|\{y ; K(x, y)>\alpha\}| \leq\left|B_{x}\left(\gamma_{x}\left(\frac{1}{\alpha}\right)\right)\right|=\frac{1}{\alpha}
$$

où $\gamma_{x}$ est l'inverse de la fonction $r \mapsto\left|B_{x}(r)\right|$. On a de même

$$
|\{x ; K(x, y)>\alpha\}|=\left|B_{x}\left[\gamma_{y}\left(\frac{1}{\alpha}\right)\right]\right|
$$

et par hypothèse

$$
\left|B_{x}(r)\right| \leq C\left|B_{y}(r)\right| \quad \text { et } \quad\left|B_{x}\left[\gamma_{y}\left(\frac{1}{\alpha}\right)\right]\right| \leq C\left|B_{y}\left[\gamma_{y}\left(\frac{1}{\alpha}\right)\right]\right|=\frac{C}{\alpha} .
$$

d'où le résultat annoncé.

Comme $|K(x, y)| \leq C$, pour tout $x$ (resp. tout $y$ fixé), $K(x, y)$ comme fonction de $y$ (resp. fonction de $x$ ) est dans $L^{p}(M)$ pour tout $1<p \leq \infty$, il s'ensuit d'après [3] que

$$
\|S(f)\|_{q} \leq C\|f\|_{p}
$$

si $1 / q=1 / p+1 / r-1=1 / p-1 / r^{\prime}$. Choisissons $r^{\prime}$ tel que $\varepsilon>1 / r^{\prime}$ et $r \gg 1$, Alors d'après ce qui a été dit précédemment la fonction de $x,\left(1+\mid B_{0}(\delta(0, x) \mid)^{-\varepsilon}\right.$, est dans $L^{r^{\prime}}(\mathrm{M})$ et

$$
\left\|\left(1+\mid B_{0}[\delta(0, x)]\right)^{-\varepsilon} S(f)\right\| \leq C\|f\|_{p}
$$

d'où le résultat de la proposition. 
2.2. Preuve du théorème 2. - La proposition suivante sera déterminante par la suite.

Proposition 6. - Soit $G=H$ (s) $G_{0}$ comme ci-dessus. On considère $z_{0}=$ $\left(x_{1}, y_{1}\right)$ avec $x_{1}$ dans $H$ et $y_{1}$ dans $G_{0}$. Alors si $\delta_{H}$ désigne la distance de contrôle associée au système de Hörmander dans $H$,

$$
\log \left[\delta_{H}\left(x_{1}, e\right)\right] \leq \beta \delta_{G}\left(z_{0}, e\right)+C
$$

où $C$ est une constante, pourvu que $\delta_{H}\left(x,,_{1}, e\right) \geq C_{2}$ et $\delta_{G_{0}}\left(e, y_{1}\right) \geq C_{2}$ où $C_{2}$ est une constante suffisamment grande.

Preuve de la proposition. - Soient $X_{1}, \ldots, X_{k}$ comme précédemment. Si $X_{j}$ est dans $\mathcal{H}$, on a $\widetilde{X}_{j} f(x, y)=\mathrm{d} f\left(x \cdot y \exp t X_{j}, y\right) / \mathrm{d} t_{\mid t=0}$ pour toute fonction $C^{\infty}$ $f: G \rightarrow \mathbb{C}$. Alors

$$
\widetilde{X}_{j} f(x, y)=\frac{\mathrm{d}}{\mathrm{d} t} f\left(x \cdot \exp \tau_{y} t X_{j}, y\right)_{\mid t=0}
$$

où $\tau_{y}$ désigne la différentielle de l'automorphisme $y \mapsto \tau_{y}: t_{y}(x)=x \cdot y$.

En particulier, si $y=\exp H_{0}$ avec $H_{0} \in \mathcal{A}_{0}$ où $\mathcal{A}_{0}$ désigne le sous-espace abélien maximal de $p_{0}$ correspondant à $\mathcal{A}_{0}^{+}$et si $X$ est dans un espace radiciel $\mathcal{H}_{i j}$, on a

$$
\widetilde{X} f\left(x, \exp H_{0}\right)=\alpha_{i j}\left(H_{0}\right) \widetilde{X}_{j \mid x}^{(1)} f\left(x, \exp H_{0}\right)
$$

où $\widetilde{X}_{j}^{(1)}$ désigne le champ des vecteurs invariant à gauche sur $H$ induit par $X_{j}$.

Soit $\psi:[0, T] \rightarrow G$ une courbe minimisante pour la distance dans $G$. On a

$$
\begin{aligned}
\psi(t) & =\left(\omega_{1}(t), \omega_{2}(t)\right) \quad \text { avec } \quad \omega_{1} \in H \text { et } \omega_{2} \in G_{0} \\
\psi(0) & =e, \quad \psi(T)=\left(x_{1}, y_{1}\right), \quad y_{1}=k_{1} \exp a k_{2}, \\
\psi^{\prime}(t) & =\left(\omega_{1}^{\prime}(t) \omega_{2}^{\prime}(t)\right)=\sum_{j=1}^{n} \psi_{j}(t) \widetilde{X}_{j} \mid \psi^{\prime}(t) \quad \text { avec } \quad \sum_{j=1}^{n}\left|\psi_{j}(t)\right|^{2}=1 .
\end{aligned}
$$

Soient $f: G \rightarrow \mathbb{C}$ une fonction $C^{\infty}$ et $1 \leq j \leq \ell$. On a

$$
\widetilde{X}_{j \mid \psi(t)} f=\frac{\mathrm{d}}{\mathrm{d} s} f\left(\psi(t) \exp s X_{j}\right)_{\mid s=0}=\frac{\mathrm{d}}{\mathrm{d} s} f\left(\omega_{1}(t), \omega_{2}(t)\right)\left(\exp s X_{j}, e\right)_{\mid s=0}
$$

où on a posé $\omega_{2}(t)=k_{1}(t) \exp a(t) k_{2}(t)$,

$$
\begin{aligned}
\left.f\left[\omega_{1}(t), \omega_{2}(t)\right)\left(\exp s X_{j}, e\right)\right] & =f\left[\left(\omega_{1}(t) \cdot \omega_{2}(t) \cdot \exp s X_{j}, \omega_{2}(t)\right]\right. \\
& =f\left[\left(\omega_{1}(t) \cdot \exp s \tau_{\omega_{2}(t)} X_{j}, \omega_{2}(t)\right],\right. \\
\frac{\mathrm{d} f}{\mathrm{~d} s}\left[\psi(t)\left(\exp s X_{j}, e\right)\right]_{\mid s=0} & =\frac{\mathrm{d}}{\mathrm{d} s} f\left[\left(\omega_{1}(t) \exp s \tau_{\omega_{2}(t)} X_{j}, \omega_{2}(t)\right]\right. \\
& =\frac{\tau_{\omega_{2}(t)} X_{j}}{\mid \psi(t)} f\left[\omega_{1}(t), \omega_{2}(t)\right] .
\end{aligned}
$$


On considère enfin une base $Y_{1}, \ldots, Y_{m_{0}}$ de $\mathcal{H}$ de vecteurs radiciels et $H_{0}$ dans $\mathcal{A} \exp H_{0} \cdot Y_{j}=\mathrm{e}^{\alpha_{\ell, j}\left(H_{0}\right)} Y_{j}$. Si $1 \leq j \leq \ell$, on a

$$
\begin{aligned}
\tau_{\omega_{2}(t)} X_{j} & =\tau_{k_{1}(t)} \tau_{a(t)} \tau_{k_{2}(t)} X_{j} \\
& =a_{j, \ell_{1}} \tau_{k_{1}(t)} \tau_{a(t)} Y_{\ell_{1}} \\
& =a_{j, \ell_{1}} \varphi_{\ell_{1}, \ell_{2}}\left(k_{2}(t)\right) \tau_{a(t)} Y_{\ell_{2}} \\
& =a_{j, \ell_{1}} \varphi_{\ell_{1}, \ell_{2}}\left(k_{2}(t)\right) \mathrm{e}^{\left.\alpha_{\ell_{2}, \ell_{3}}(a t)\right)} \varphi_{\ell_{1}, \ell_{2}^{\prime}}\left(k_{2}(t)\right) Y_{\ell_{2}^{\prime}} \\
& =a_{j, \ell_{1}} \varphi_{\ell_{1}, \ell_{2}}\left(k_{2}(t)\right) a^{\ell_{2}^{\prime}, \ell_{3}^{\prime}} \mathrm{e}^{\left.\alpha_{\ell_{2}, \ell_{3}}(a t)\right)} \varphi_{\ell_{1}, \ell_{2}^{\prime}}\left(k_{2}(t)\right) X_{\ell_{3}^{\prime}}^{0}
\end{aligned}
$$

où l'on a utilisé la sommation muette. Par ailleurs si $\ell+1 \leq j \leq k$, on a

$$
\left.\left(^{*}\right) \quad \widetilde{X}_{j}\right|_{\psi(t)} f=\frac{\mathrm{d}}{\mathrm{d} s} f\left(\omega_{1}(t), \omega_{2}(t) \exp s X_{j}\right)_{s=0}=\widetilde{X}_{j}^{0} f
$$

Mais $\psi^{\prime}(t)=\left(\omega_{1}^{\prime}(t), \omega_{2}^{\prime}(t)\right) ;$ l'expression de $\widetilde{X}_{j} \mid \psi(t)$ pour $1 \leq j \leq \ell$ prend la forme

D'après $(*)$,

$$
a^{\ell_{2}^{\prime}, \ell_{3}^{\prime}} a_{j, \ell_{1}} \varphi_{\ell_{1}, \ell_{2}}\left(k_{1}(t)\right) \varphi_{\ell_{2}, \ell_{2}^{\prime}}\left(k_{2}(t)\right) \mathrm{e}^{\alpha \ell_{2}, \ell_{3}}(a(t)) \widetilde{X}_{\ell_{3}^{\prime}}^{0(1)} .
$$

$$
\omega_{1}^{\prime}(t)=\psi_{j}(t) a^{\ell_{2}^{\prime}, \ell_{3}^{\prime}} a_{j, \ell_{1}} \mathrm{e}^{\alpha_{\ell_{2}, \ell_{3}}(a(t))} \varphi_{\ell_{1}, \ell_{2}}\left(k_{1}(t)\right) \varphi_{\ell_{2}, \ell_{2}^{\prime}}\left(k_{2}(t)\right) \widetilde{X}_{\ell_{3}^{\prime}}^{0(1)} .
$$

Si $\mathcal{X}$ désigne la projection sur le plan engendré par $\left(\widetilde{X}_{1}, \ldots, \widetilde{X}_{\ell_{1}}\right)$

$$
\begin{aligned}
\omega_{1}^{\prime}(t) & =\psi_{j}(t) a^{\ell_{2}^{\prime}, \ell_{3}^{\prime}} a_{j, \ell_{1}} \mathrm{e}^{\alpha_{\ell_{2}, \ell_{3}}(a(t))} \varphi_{\ell_{1}, \ell_{2}}\left(k_{1}(t)\right) \varphi_{\ell_{2}, \ell_{2}^{\prime}}\left(k_{2}(t)\right) \mathcal{X}\left(\widetilde{X_{\ell_{3}^{\prime}}^{0}}{ }^{(1)}\right. \\
& =\psi_{j}(t) a^{\ell_{2}^{\prime}, \ell_{3}^{\prime}} a_{j, \ell_{1}} \mathrm{e}^{\alpha_{\ell_{2}, \ell_{3}}(a(t))} \varphi_{\ell_{1}, \ell_{2}}\left(k_{1}(t)\right) \varphi_{\ell_{2}, \ell_{2}^{\prime}}\left(k_{2}(t)\right) U_{\ell_{3}^{\prime}, \ell_{4}} \widetilde{X}_{\ell_{4}}^{(1)}
\end{aligned}
$$

soit

$$
\omega_{\ell_{4}}^{\prime}=\psi_{j}(t) a^{\ell_{2}^{\prime}, \ell_{3}^{\prime}} a_{j, \ell_{1}} \mathrm{e}^{\alpha_{\ell_{2}, \ell_{3}}(a(t))} \varphi_{\ell_{1}, \ell_{2}}\left(k_{1}(t)\right) \varphi_{\ell_{2}, \ell_{2}^{\prime}}\left(k_{2}(t)\right) U_{\ell_{3}, \ell_{4}} .
$$

Comme $K_{0}$ est compact, $\sup _{k \in k_{0}}\left|\varphi_{\ell_{1}, \ell_{2}}\left(k_{1}(t)\right)\right|=a_{1}$ est fini. Mais

$$
\left|\psi_{j}(t)_{a_{j}, \ell_{1}}\right| \leq\left(\sum\left|a_{j, \ell_{1}}\right|^{2}\right)^{1 / 2}=a_{2}, \quad \sup \left|U_{\ell_{3}, \ell_{4}}\right|=a_{3}
$$

et

$$
\left({ }^{* *}\right) \quad\left|\omega_{\ell_{4}}^{\prime}\right|(t) \leq a_{1}^{2} a_{2}^{2} a_{3} \mathrm{e}^{\alpha_{\ell_{2}, \ell_{3}}(a(t))} .
$$

Puisque $\psi^{\prime}(t)=\sum_{j=1}^{m} \theta_{j}(t) \widetilde{X}_{j \mid \psi(t)}=\omega_{1}^{\prime}(t)+\omega_{2}^{\prime}(t)$, on a

$$
\omega_{1}^{\prime}(t)=\left.\sum_{j=1}^{\ell} \omega_{j, 1}^{\prime}(t) \widetilde{X}_{j}^{(1)}\right|_{\omega(t)}
$$

La majoration $(* *)$ montre que

$$
\left|\omega_{j}^{\prime}(t)\right| \leq c \mathrm{e}^{\|\alpha\| \cdot\|a(t)\|}
$$

où $\|a(t)\|$ est la norme donnée par la forme de Killing. 
On voudrait montrer que

$$
\|a(t)\| \leq \gamma^{2} t
$$

Les $X_{i}, \ell+1 \leq i \leq k$ engendrent un système de Hörmander sur $G_{0}$ d'où une distance associée notée $\delta_{1}$. Alors $\delta_{1}\left(e_{2}, \omega_{2}(t)\right) \leq \delta(e, \psi(t)) \leq \int_{0}^{t} \mathrm{~d} s=t$.

On désigne l'élément neutre de $G$ par $e=\left(e_{1}, e_{2}\right)$. Soit $\eta(s)$ une courbe dans $G_{0}$ qui joint $e_{2}$ à $\omega_{2}(t)$ telle que

$$
\begin{gathered}
\eta^{\prime}(s)=\sum_{i=\ell+1}^{k} \eta_{i}^{\prime}(s) \widetilde{X}_{i \mid \eta(s)} \quad \text { avec } \quad \sum\left|\eta_{i}^{\prime}(s)\right|^{2}=1 \\
\eta(\tau)=\omega_{2}(t), \quad \delta_{1}\left(e_{2}, \omega_{2}(t)\right)=\tau \\
\eta(s)=\exp X(s) k(s) .
\end{gathered}
$$

Comme

$$
\begin{aligned}
& \omega_{2}(t)=k_{1}(t) \exp a(t) k_{2}(t)=k_{1}(t) \exp a(t) k_{1}^{-1}(t) k_{1}(t) k_{2}(t), \\
& =\exp \operatorname{Ad}\left(k_{1}(t)\right) a(t) k_{1}(t) k_{2}(t), \\
& X(\tau)=\operatorname{Ad}\left(k_{1}(t)\right) a(t), \quad k(\tau)=k_{1}(t) k_{2}(t) \\
& \eta^{\prime}(s)=\left.\operatorname{Ad} \overline{\left(k^{-1}(s)\right) X^{\prime}}(s)\right|_{\exp X(s) k(s)}+\widetilde{Z}(s)
\end{aligned}
$$

où $\widetilde{Z}$ provient des champs sur $K_{0}$, on a

$$
\begin{aligned}
\left\|\eta^{\prime}(s)\right\|_{1}^{2} & =\sum_{i=\ell+1}^{k}\left|\eta_{i}(s)\right|^{2} \cdot\left\|\widetilde{X}_{i} \mid \eta(s)\right\|^{2} \\
& =\left\|\left.\operatorname{Ad} \overline{\left(k^{-1}(s)\right) X^{\prime}}(s)\right|_{\exp X(s) k(s)}+\widetilde{Z}(s)\right\|_{1}^{2} \\
& =\left\|\operatorname{Ad}\left(k^{-1}(s)\right) X^{\prime}(s)\right\|_{1}^{2}+\|\widetilde{Z}(s)\|_{1}^{2}
\end{aligned}
$$

et $\left\|X^{\prime}(s)\right\|_{1} \leq\left\|\operatorname{Ad}(k(s)) \eta_{0}^{\prime}(s)\right\|_{1}$ avec $\eta_{0}^{\prime}(s)=\sum_{i=\ell+1}^{k} \eta_{i}(s) X_{i}$, d'où

$$
\left\|X^{\prime}(s)\right\|_{1} \leq \sup _{k \in K_{0}}\|\operatorname{Ad}(k)\| \cdot\left\|\eta_{0}^{\prime}\right\|_{1}=\gamma_{0} .
$$

Alors

$$
\begin{array}{r}
\|X(\tau)\|_{1} \leq \int_{0}^{\tau}\left\|X^{\prime}(s)\right\|_{1} \mathrm{~d} s \leq \gamma_{0} \tau=\gamma_{0} \delta_{1}\left(e, \omega_{2}(t)\right) \\
a(t)=\operatorname{Ad}\left(k^{-1}(t)\right) X(\tau) \\
\|a(t)\|_{1} \leq \gamma_{0}\|X(t)\|_{1} \leq \gamma_{0}^{2} \delta_{1}\left(e, \omega_{2}(t)\right) \leq \gamma_{0}^{2} t .
\end{array}
$$


2.3. Preuve de la proposition 3. - Écrivons

$$
\begin{aligned}
\frac{1}{|B(r)|} \int \chi_{r}(h g) \mathrm{d} h & =\frac{1}{|B(r)|} \int_{\delta(h g, e) \leq 1} \chi_{r}(h g) \mathrm{d} h+\frac{1}{|B(r)|} \int_{\delta(h g, e) \geq 1} \chi_{r}(h g) \mathrm{d} h \\
& =F_{1}(g)+F_{2}(g) .
\end{aligned}
$$

La norme de $F_{1}$ comme opérateur de convolution de $L^{p}\left(G_{0}\right)$ est dominée par $c /|B(r)|$; par conséquent, il suffit d'estimer celle de $F_{2}$. Pour tout $\varepsilon>0$ fixé,

$$
\begin{aligned}
F_{2}(g) & =\frac{1}{|B(r)|^{\varepsilon}} \int_{\delta(h g, e) \geq 1} \frac{\chi_{r}(h g) \mathrm{d} h}{|B(r)|^{1-\varepsilon}} \\
& \leq \frac{C}{|B(r)|^{\varepsilon}} \int_{\delta(h g, e) \geq 1} \frac{\chi_{r}(h g) \mathrm{d} h}{c_{1}+\left|B^{1-\varepsilon}(\delta(h g, e))\right|} \\
& =\frac{C}{|B(r)|^{\varepsilon}} F_{2, \varepsilon}(g) \leq c \mathrm{e}^{-\beta_{\varepsilon} r} F_{2, \varepsilon}(g)
\end{aligned}
$$

où $C_{1}$ est une constante qu'on choisira comme il convient.

Les calculs pour $F_{2, \varepsilon}$ sont les mêmes que pour la fonction $E_{1}$ que nous allons définir ci-dessous. On va d'ailleurs voir que

$$
\sup _{k_{1}, k_{2}} F_{2, \varepsilon}\left(k_{1} \exp a k_{2}\right) \leq F_{2, \varepsilon}(\exp a) \quad \text { avec de bonnes propriétés. }
$$

\section{Suite de la preuve du théorème 2}

Soit

$$
E(x)=\frac{1}{|B[\delta(0, x)]|} \mathbf{1}_{B_{1}^{c}}(x) \simeq \frac{1}{|B(\delta(x, 0))|+C}
$$

où $\mathbf{1}_{B_{1}^{c}}(x)$ est la fonction caractéristique du complémentaire de la boule de rayon 1 centrée à l'origine de G. D'après [6] et le théorème de Kunze-Stein [6], il suffit de voir que la fonction

$$
E_{1}(x)=\int_{H} E(h x) \mathrm{d} h
$$

est dans $L^{p}\left(G_{0}\right)$ pour une valeur de $p$ inférieure à celle de l'énoncé. En effet comme $H$ est moyennable, $E$ donne lieu à un opérateur borné sur $L^{p_{1}}(G)$ si et seulement si $E_{1}$ donne lieu à un opérateur borné de $L^{p_{1}}\left(G_{0}\right)$ (ceci résulte de [6]) et il en est ainsi si $E_{1}$ est dans $L^{p}\left(G_{0}\right)$.

On veut d'abord estimer $E_{1}(x)$ pour $\delta_{1}(e, \dot{x})$ très grand. On pose

$$
x=k \exp a k_{1}
$$

et l'on voit que

$$
(* * *) \quad \delta\left(h k \exp a k_{1}, e\right) \geq \delta(h k \exp a, e)-C
$$


car par hypothèse $\delta_{1}(e, \dot{x})$ est très grand comme c'est une distance quotient $\delta\left(h k \exp a k_{1}, e\right) \geq \delta_{1}(e, \dot{x})$ qui est très grand. Alors

$$
\frac{1}{\left|B\left[\delta\left(h k \exp a k_{1}, e\right)\right]\right|} \leq \frac{C_{1}}{|B[\delta(h k \exp a, e)]|} .
$$

En effet, soient $r, s>0$; alors $B(r+s)=\bigcup_{y \in B(r)} B_{y}(s)$. Si $Y$ est un sousensemble fini de $B(r)$ dont deux points $y_{1}, y_{2}$ avec $y_{1} \neq y_{2}$ satisfont l'inégalité $\delta\left(y_{1}, y_{2}\right)>b$ où $b$ est une constante positive

$$
\bigcup_{y \in Y} B_{y}\left(\frac{1}{2} b\right) \subset B\left(r+\frac{1}{2} b\right)
$$

et $\operatorname{Card}(Y)\left|B_{y}\left(\frac{1}{2} b\right)\right|=\operatorname{Card} Y C_{b} \leq\left|B\left(r+\frac{1}{2} b\right)\right|$. Soit $Y \subset B(r)$ fini, maximal pour la propriété : tous les points $y_{1}, y_{2}$ de $Y$ avec $y_{1} \neq y_{2}$ satisfont $\delta\left(y_{1}, y_{2}\right)>b$. D'après la maximalité de $Y$, on a l'inclusion $B(r+s) \subset \bigcup_{y \in Y} B(y, s+b)$ et

$$
|B(r+s)| \leq \operatorname{Card}(Y)|B(s+b)| \leq C_{b}^{-1}\left|B\left(r-\frac{1}{2} b\right)\right| \cdot|B(s+b)| .
$$

Si l'on pose $r+\frac{1}{2} b=r_{1}$ et $s-\frac{1}{2} b=s_{1}$, on a

$$
\left|B\left(r_{1}-s_{1}\right)\right| \leq C_{b}^{-1} B\left(r_{1}\right) B\left(s_{1}+\frac{3}{2} b\right)
$$

pour $r_{1}>\frac{1}{2} b$ et $s \geq-\frac{1}{2} b$; en particulier pour $s_{1} \geq 0$, on trouve avec l'inégalité $\delta(h k \exp a, e) \leq c+\delta\left(h k \exp a k_{1}, e\right)$

$$
\begin{aligned}
|B[\delta(h k \exp a, e)]| & \left.\leq \mid B\left(\delta\left(h k \exp a k_{1}, e\right)\right)+c\right) \\
& \leq C_{b}^{-1} \mid B\left[\left(\delta(h k \exp a, e]|\cdot| B\left(c+\frac{3}{2} b\right) \mid\right.\right.
\end{aligned}
$$

d'où l'inégalité ci-dessus.

Soit $r$ très grand; on veut estimer $\left|B_{e}(r)\right|=|B(r)|$. Soit $\gamma_{1}=\sup _{k \in K_{0}} \delta(k, e)$; si $\delta(g, e)$ est très grand et que $g=k_{1} \exp X k_{2}, k_{i} \in K, X$ dans $a, g \in G_{0}$

$$
\delta(\exp X, e)-2 \gamma_{1}<\delta(g, e) \leq \delta(\exp X, e)+2 \gamma_{1}
$$

Par ailleurs $\delta(\exp X, e) \leq \delta_{G_{0}}\left(\exp X, e_{1}\right)$ où $\delta_{G_{0}}$ est la distance induite sur $G_{0}$.

Comme

$$
\delta_{G_{0}}(\exp X, e) \leq\|X\| \delta(g, e) \leq\|X\|+\gamma_{1}
$$

on a

$$
\begin{gathered}
B^{\prime}=\left\{g=k_{1} \exp X k_{2},\|X\| \leq r-1-2 \gamma\right\}, \\
B^{\prime} \cdot B(1) \subset B(r), \quad B^{\prime} \cdot B(1)=\left\{(g \cdot x, g \cdot y), g \in B^{\prime}, \delta(x, y, e) \leq 1\right\}, \\
\int_{B_{r}} \mathrm{~d} x \geq \int_{B^{\prime} \cdot B(1)} \mathrm{d} x .
\end{gathered}
$$


La trace de la boule $B(1)$ sur $H$ sera notée $B^{\prime \prime}(1)$. Alors

$$
\begin{gathered}
B^{\prime} \circ B^{\prime \prime}(1) \subset B(r) \quad \text { et } \quad B^{\prime} \circ B^{\prime \prime}(1)=\left\{(g, x), g \in B^{\prime}, x \in B^{\prime \prime}(1)\right\}, \\
\int_{B_{r}} \mathrm{~d} x \geq \int_{B^{\prime}} \mathrm{d} g \int_{g B^{\prime \prime}(1)} \mathrm{d} u .
\end{gathered}
$$

Quitte à diminuer l'intégrale ci-dessus, on peut trouver des boules $D_{j, n}^{0}$ dans $H^{i}$ de petit rayon, pour une bonne m étrique $K$ invariante, telle que pour tout $x_{j} \subset D_{j, n}^{0}$, $\exp x_{i} \in B^{\prime}(1)$ et $x=\prod_{j=1}^{m} \exp x_{j} \in B^{\prime \prime}(1)$. Si $a \in \mathfrak{A}^{+}$, alors $\exp a \cdot x=\prod_{j=1}^{m} \exp \operatorname{Ad} a x_{j}$.

Si $x_{j}$ est dans l'espace radiciel d'indice $n, x_{j}$, on l'indexe $x_{n, j}$ et on a

$$
\prod_{j=1}^{m} \exp \left[\mathrm{e}^{\alpha_{n, j}(a)} x_{n, j}\right]
$$

Si $k \in K$, on a

$$
k \cdot \prod_{j=1}^{m} \exp \mathrm{e}^{\alpha_{n, j}(a)} x_{n, j}=\prod_{j=1}^{m} \exp \mathrm{e}^{\alpha_{n, j}(a)} \operatorname{Ad} k \cdot x_{n, j} .
$$

Comme on a supposé qu'il existe des sous-ensembles $D_{j, n}$ dans l'espace radiciel $H_{j, n}$ tels que pour tout $x_{j, n} \in D_{j, n}$

$$
\left\|x_{j, n}\right\| \leq 1, \quad\left[x_{j, n}, a\right]=\alpha_{j, n}(a) x_{j, n} \quad \text { avec } \alpha_{j, n}(a) \geq 0
$$

on a

$$
\begin{aligned}
& \int_{\prod_{j=1}^{n} \mathrm{~d} x \cdot \prod_{n=1}^{m_{j}} \exp D_{j, n}^{+}}>C_{1}>0 \\
& \int_{B^{\prime}} \mathrm{d} g \int_{g B^{\prime \prime}(1)} \mathrm{d} x \geq \int_{\substack{a \in a^{+} \\
\|a\| \leq r-1-2 \gamma}} \prod_{\alpha \in \Sigma^{+}} S h^{m_{\alpha}}(a) \\
& \times \int_{K} \mathrm{~d} k \int_{k \cdot \prod_{j=1}^{n} K \cdot \prod_{n=1}^{m_{j}} \exp ^{\alpha_{j, n}(a)} D_{j, n}^{+}}^{\mathrm{d} x \mathrm{~d} a} \\
& =\int_{\substack{a \in a^{+} \\
\|a\| \leq r-1-2 \gamma}} \prod_{\alpha \in \Sigma^{+}} S h^{m_{\alpha}}(a) \mathrm{d} a \int_{\prod_{j=1}^{n} K \cdot \prod_{n=1}^{m_{j}} \operatorname{exp~e}^{\alpha_{j, n}(a)} D_{j, n}^{+}} \mathrm{d} x \\
& =\int_{\substack{a \in a^{+} \\
\|a\| \leq r-1-2 \gamma}} \prod_{\alpha \in \Sigma^{+}} S h^{m_{\alpha}}(a) \mathrm{d} a \mathrm{e}^{\Sigma \alpha_{j, n}(a)} \int_{\prod_{j=1}^{n} K \cdot \prod_{n=1}^{m_{j}} \exp D_{j, n}^{+}}^{\mathrm{d} x} \\
& \geq C_{0} \int_{\substack{a \in a^{+} \\
\|a\| \leq r-1-2 \gamma}} \prod_{\alpha \in \Sigma^{+}} S h^{m_{\alpha}}(a) \mathrm{e}^{\Sigma m_{i j} \alpha_{i j}(a)} \mathrm{d} a \\
& \geq C_{0} \int_{\substack{a \in a^{+} \\
\alpha(a) \geq \sqrt{2} \\
\|a\| \leq r-1-2 \gamma}} \mathrm{e}^{2 \rho(a)+\theta(a)} \mathrm{d} a \geq C^{\mathrm{te}} \mathrm{e}^{r\|2 \rho+\theta\|} r^{\ell} .
\end{aligned}
$$

TOME $135-2007-\mathrm{N}^{\mathrm{O}} 3$ 
Nous sommes donc amenés à estimer

$$
\begin{gathered}
\int_{H} \frac{\mathbf{1}_{B^{c}}(h k \exp a) \mathrm{d} h}{\mid B\{\delta(h k \exp a), e]\} \mid+C_{1}}=\int_{H} \frac{\mathrm{d} h}{\left|B\left[\delta\left\{\left(k k^{-1} h k \exp a\right), e\right\}\right]\right|+C_{1}} \\
\leq \int_{H} \frac{\mathrm{d} h^{\prime}}{\left.\mid B\left(\delta\left(k h^{\prime} \exp a\right), e\right)\right) \mid+C_{1}} \\
\leq C_{2} \int_{H} \frac{\mathrm{d} h}{|B(\delta(h \exp a, e))|+C_{1}} \quad \text { (d'après (***)). }
\end{gathered}
$$

Si $\delta_{H}\left(h, e_{2}\right) \leq 1 \cdot \delta(h, e) \leq 1$, d'après [9] et

$$
\begin{aligned}
\int_{\delta_{H}\left(h, e_{2}\right) \leq 1} & \frac{\mathrm{d} h}{\mid B(\delta(h \exp a), e)) \mid+C_{1}} \\
\leq C \frac{1}{\mid B[\delta(\exp a), e)] \mid+C_{1}} & =F_{0}^{\prime}(\exp a) .
\end{aligned}
$$

La restriction de $\delta$ à $G_{0}$ est une distance invariante à gauche; par conséquent, $F_{0}^{\prime}$ est dans $L^{p}\left(G_{0}\right)$ pour tout $1<p \leq \infty$ et

$$
\int_{H} \frac{\mathbf{1}_{B^{c}}(h g k) \mathrm{d} h}{|B(\delta(h g k, e))|+C} \leq C \int_{H} \frac{\mathrm{d} h}{|B[\delta(h g, e)]|+C_{1}} .
$$

Pour estimer $E_{1}$, il suffit donc d'estimer

$$
\int_{\delta_{H}(h, e) \geq 1} \frac{\mathrm{d} h}{|B\{\delta(h \exp a, e)\}|+C}=F_{0}^{\prime}(a) .
$$

Mais si $\varepsilon>0$, on a

$$
\begin{aligned}
\tau(a)= & \int_{\delta_{H}\left(h, e_{2}\right) \geq 1} \frac{\delta^{\ell}(h \exp a, e) \delta^{-\ell}(h \exp a, e) \mathrm{e}^{2\|\rho\|(1-\varepsilon) \delta\left(h \exp a, e_{2}\right)}}{\mathrm{e}^{2 \mid \rho \|(1-\varepsilon) \delta\left(h \exp a, e_{2}\right)}\left|B\left(\delta\left(h \exp a, e_{2}\right)\right)\right|+C} \\
\leq \delta_{G_{0}}^{-\ell}(\exp a, e) \mathrm{e}^{-2\|\rho\|(1-\varepsilon) \delta_{G_{0}}(\exp a, e)} & \\
& \times \int_{\delta_{H}(e, h) \geq 1} \frac{\mathrm{e}^{2 \mid \rho \|(1-\varepsilon) \delta\left(h \exp a, e_{2}\right)}}{|B(\delta(h \exp a, e))|+C} \mathrm{~d} h .
\end{aligned}
$$

Pour évaluer la dernière intégrale, on va utiliser l'estimation

$$
\begin{aligned}
& \mathrm{e}^{2\|\rho\|(1-\varepsilon) \delta(h \exp a, e)} \mathrm{e}^{-\|2 \rho+\theta\| \delta(h \exp a, e)} \delta^{\ell}(h \exp a, e) \\
& \leq \mathrm{e}^{-\left\{\|2 \rho+\theta\|-2\|\rho\|\left(1-\varepsilon^{\prime}\right)\right\} \delta(h \exp a, e)}, \quad \varepsilon \approx \varepsilon
\end{aligned}
$$

puisque $\|2 \rho+\theta\|>2\|\rho\|(1-\varepsilon)$.

Mais d'après la proposition 1 , on a

$$
\begin{aligned}
\mathrm{e}^{2 \mid \rho \|(1-\varepsilon) \delta(h \exp a, e)} \mathrm{e}^{-\|2 \rho+\theta\| \delta(h \exp a, e)} \delta^{\ell}(h \exp a, e) & \\
& \leq C\left(\delta_{H}\left(h, e_{2}\right)\right)^{-\left\{\|2 \rho+\theta\|-2\|\rho\|\left(1-\varepsilon^{\prime}\right)\right\} / \beta} .
\end{aligned}
$$


Mais par hypothèse

$$
\left|\left\{h ; \delta_{H}^{-\gamma}\left(h, e_{2}\right)>\alpha\right\}\right|=\left|B\left(\alpha^{-1 / \gamma}\right)\right| \leq C\left(\frac{1}{\alpha}\right)^{D / \gamma}
$$

et

$$
\begin{array}{r}
\int_{\delta_{H}(e, h) \geq 1} \frac{\mathrm{d} h}{\left\{\delta_{H}\left(h, e_{2}\right)\right\}^{\left.\|2 \rho+\theta\|-2\|\rho\|\left(1-\varepsilon^{\prime}\right)\right\} / \beta}} \\
\leq C \int_{0}^{1} \frac{d \alpha}{\alpha^{D \beta /\left(\|2 \rho+\theta\|-2\|\rho\|\left(1-\varepsilon^{\prime}\right)\right)}}<\infty
\end{array}
$$

d'où la finitude de l'intégrale si $p_{1}(\varepsilon)=1 /(1-\varepsilon)$. Il s'ensuit que

$$
F_{2,0}\left(k \exp a k^{\prime}\right) \leq c \delta_{G_{0}}^{-\ell}(\exp a, e) \mathrm{e}^{-2\|\rho\|(1-\varepsilon) \delta_{G_{0}}(\exp a, e)} .
$$

Comme on ne s'intéresse qu'aux a grands, $F_{2,0}$ est dans $L^{p_{1}-\varepsilon_{0}}\left(G_{0}\right)$ et d'après [6], l'opérateur de convolution associé est borné sur $L^{p}\left(G_{0}\right.$ et d'après [5], $E$ donne lieu à un opérateur borné sur $L^{p}(G)$.

\subsection{Démonstration du théorème 4}

1) Comme la courbure de Ricci est $\geq-b^{2}$, si l'on note $\Delta$ le laplacien de $M$, on a d'après le théorème de comparaison (voir [1])

$$
\frac{1}{n-1} \Delta r \leq \Delta_{b} r_{b}
$$

où $r$ désigne la fonction distance sur $M$ d'un point distingué et $r_{b}$ la fonction distance sur la variété simplement connexe hyperbolique de courbure de Ricci $-b^{2}$ et de dimension $n$. Si $\theta(s, w)$ désigne la densité de volume en coordonnées polaires exponentielles au point $x$ de $M$, on en déduit que

$$
\frac{1}{n-1} \theta^{\prime}(s, w) \leq c \theta(s, w)
$$

pour $s \geq 1$, et tout point $w$ de la sphère-unité de $T_{x} M$. Alors

$$
\begin{gathered}
\int_{1}^{r} \theta^{\prime}(s, w) \mathrm{d} s \leq c \int_{0}^{r} \theta(s, w) \mathrm{d} s \\
\int_{\Sigma_{n-1}}[\theta(r, w)-\theta(1, w)] \mathrm{d} w \leq c \int_{0}^{r} \int_{\Sigma_{n-1}} \theta(s, w) \mathrm{d} s \mathrm{~d} w
\end{gathered}
$$

si $r$ est assez grand, $\frac{1}{10} \int_{\Sigma_{n-1}} \theta(r, w) \mathrm{d} w>\int_{\Sigma_{n-1}} \theta(1, w) \mathrm{d} w$ alors

$$
\int_{\Sigma_{n-1}} \theta(r, w) \mathrm{d} w \leq c \int_{0}^{r} \int_{\Sigma_{n-1}} \theta(s, w) \mathrm{d} s \mathrm{~d} w .
$$

Il s'ensuit que pour $r$ assez grand,

$$
\int_{\Sigma_{n-1}} \theta(r, w) \mathrm{d} w \leq c\left|B_{r}(x)\right|
$$

TOME $135-2007-\mathrm{N}^{\mathrm{O}} 3$ 
où $B_{r}(x)$ désigne la boule riemannienne de centre $x$ et de rayon $r$ et $\left|B_{r}(x)\right|$ son volume.

On commence la preuve du théorème comme pour celle de [1]. En effet

$$
\begin{aligned}
f^{*}(x) \leq \operatorname{Sup}_{r \leq r_{0}} & \frac{1}{\left|B_{r}(x)\right|} \int_{B_{r}(x)}|f(y)| \mathrm{d} \sigma(y) \\
& \quad+\operatorname{Sup}_{r \geq r_{0}} \frac{1}{\left|B_{r}(x)\right|} \int_{B_{r}(x)}|f(y)| \mathrm{d} \sigma(y) .
\end{aligned}
$$

On choisit $r_{0}$ très grand pour que l'inégalité

$$
\frac{1}{10} \int_{\Sigma_{n-1}} \theta(r, w) \mathrm{d} w>\int_{\Sigma_{n-1}} \theta(1, w) \mathrm{d} w
$$

soit vérifiée pour tout $r>r_{0}$.

On continue comme précédemment et l'on s'intéresse à

$$
\begin{aligned}
f_{2}^{0}(x) & =\operatorname{Sup}_{r \geq r_{0}} \frac{1}{\left|B_{r}(x)\right|} \int_{B_{r}(x)}|f(y)| \mathrm{d} \sigma(y) \\
& \leq \int_{M} \operatorname{Inf}\left(1, \frac{1}{\left|B_{\delta(x, y)}(x)\right|}\right)|f(y)| \mathrm{d} \sigma(y) \\
& \leq \int_{\delta(x, y) \leq r_{0}}|f(y)| \mathrm{d} \sigma(y)+\int_{\delta(x, y) \geq r_{0}} \frac{1}{B_{\delta(x, y)}(x)}|f(y)| \mathrm{d} \sigma(y) \\
& ? ? ? \int_{M}\left|\int_{\delta(x, y) \leq \mathbb{C}}\right| f(y)|\mathrm{d} \sigma(y)|^{p} \mathrm{~d} \sigma(x) \leq C\|f\|_{p}^{p} .
\end{aligned}
$$

Car $M$ est à géométrie bornée. Il ne nous reste plus que la fonction

$$
f_{2}(x)=\int_{\delta(x, y) \geq r_{0}} \frac{1}{\left(\left|B_{\delta(x, y)}(x)\right|\right.}|f|(y) \mathrm{d} \sigma(y) .
$$

D'après la première partie le noyau intéressant à étudier est

$$
K^{-1}(\delta(x, y))=\int_{\Sigma_{n-1}} \theta[\delta(x, y), w] \mathrm{d} w \mathbf{1}_{\delta(x, y) \geq r_{0}}
$$

puisque

$$
f_{2}(x) \leq \int_{M} K(x, y)|f(y)| \mathrm{d} \sigma(y)
$$

On se propose d'estimer $K(x, y)$. On considère un système de coordonnées polaires au point $x$ dans $T_{x} M$. On note $(r, w)$ un système de coordonnées polaires en $x_{0}, r=\delta(x, y)$ le volume de la boule de centre $x$ de rayon $r$ vaut 
$\left|B_{r}(x)\right|=\int_{0}^{r} \int_{\Sigma_{n-1}^{\theta(r, w) \mathrm{d} w}}$ et l'aire de la sphère $\mathrm{e}^{\varphi(r)}=\int_{\Sigma_{n-1}^{\theta(r, w) \mathrm{d} w}}$ pour $\delta(x, y)$ suffisamment grand, le laplacien de la fonction $\psi_{x}(y)=\mathrm{e}^{-\varphi_{x}(\delta(x, y))}$ vaut

$$
\Delta_{y} \psi_{x}(y)=\left[-\frac{\mathrm{d}^{2} \phi_{x}(r)}{\mathrm{d} r^{2}}+\left(\frac{\mathrm{d} \phi_{(r)}}{\mathrm{d} r}\right)^{2}-\frac{\theta_{x}^{\prime}(r, w)}{\theta_{x}(r, w)} \frac{\mathrm{d} \phi_{x}(r)}{\mathrm{d} r}\right] \mathrm{e}^{-\varphi_{x}[\delta(x, y)]} .
$$

Mais $\theta_{x}^{\prime}(r, w) / \theta(r, w)=H(r, w)$ où $H(r, w)$ est la courbure moyenne de la sphère centrée en $x$ et de rayon $r$ évaluée au point de coordonnées polaires $(r, w)$.

Il existe par hypothèse $\sigma$ telle que $0<\sigma<\sigma_{0}$ et

$$
\left[-\frac{d^{2} \phi_{x}(r)}{\mathrm{d} r^{2}}+\left[\frac{d \phi_{x}(r)}{\mathrm{d} r}\right]^{2}-\tau \frac{d \phi_{x}(r)}{\mathrm{d} r}+\sigma^{2}\right] \mathrm{e}^{-\phi_{x}(r)} \geq 0 .
$$

Alors

$$
\left[-\frac{\mathrm{d}^{2} \varphi_{x}(r)}{\mathrm{d} r^{2}}+\left[\frac{\mathrm{d} \varphi_{x}(r)}{\mathrm{d} r^{2}}\right]^{2}-H(r, w) \frac{\mathrm{d} \varphi_{x}(r)}{\mathrm{d} r}+\sigma^{2}\right] \mathrm{e}^{-\varphi_{x}(r)} \geq 0
$$

et la fonction $\psi_{x}(y)=\mathrm{e}^{-\varphi_{x}(\delta(x, y)}$ est $\Delta+\sigma$ sous harmonique.

Mais si $f$ est une fonction $M \rightarrow \mathbb{C}, C^{\infty}$ à support compact, $f\left(x_{0}\right)=0$

$$
\begin{aligned}
& \int_{0}^{\infty}|f(r, w)|^{2} \theta(r, w) \mathrm{d} r \leq \frac{1}{\sigma} \int_{0}^{\infty}|f(r, w)|^{2} \theta^{\prime}(r, w) \mathrm{d} r \\
& \frac{1}{\sigma} \int_{0}^{\infty}\left|\frac{\partial f}{\partial r}(r, w)\right| \cdot|f(r, w)| \theta(r, w) \mathrm{d} r \\
& \quad \leq \frac{1}{\sigma}\left(\int_{0}^{\infty}\left|\frac{\partial f}{\partial r}(r, w)\right|^{2} \theta(r, w) \mathrm{d} r\right)^{1 / 2} \cdot\left(\int_{0}^{\infty}|f(r, w)|^{2} \theta(r, w) \mathrm{d} r\right)^{1 / 2}, \\
& \int_{0}^{\infty} \int_{\Sigma_{n-1}}|f(r, w)|^{2} \theta(r, w) \mathrm{d} r \mathrm{~d} w \leq \frac{1}{\sigma_{0}^{2}} \int_{0}^{\infty} \int_{\Sigma_{n-1}}\left|\frac{\partial f}{\partial r}(r, w)\right|^{2} \mathrm{~d} r \mathrm{~d} w
\end{aligned}
$$

et $\|f\|_{2}^{2} \leq-\frac{1}{\sigma_{0}^{2}}\langle\Delta f, f\rangle$

$$
\left\langle\left(-\Delta-\sigma_{0}^{2}\right) f, f\right\rangle \geq 0
$$

et si $\varepsilon>0,\left\langle-\Delta-\left(\sigma^{2}\right) f, f\right\rangle \gg\left(\sigma^{2}-\sigma^{2}\right)\|f\|_{2}^{2}$.

Par conséquent

$$
\Delta+\sigma^{2} \text { est inversible sur } L^{2}(M)
$$

d'où l'existence d'une fonction de Green pour $\Delta+\sigma^{2}: G_{\sigma}$.

On peut appliquer le principe du maximum à $\psi$ et $G_{\sigma}$ avec l'opérateur elliptique $\Delta+\sigma^{2}$. Malheureusement la constante $\sigma$ est positive et on ne peut l'appliquer brutalement.

a) Comme $M$ est à géométrie bornée pour $\delta(x, y)=r_{0}>0, C_{\sigma}^{-1}<$ $G_{\sigma}(x, y) \leq C_{\sigma}$ où $C_{\sigma}$ est une constante finie qui ne dépend ni de $x$, ni de $y$. 
b) On considère l'opérateur

$$
\left(G_{\sigma}^{x}\right)^{-1}(\Delta+\sigma) G_{\sigma}^{x}=\Delta+\nabla \log G_{\sigma}^{x}=\Delta_{\sigma}^{x}
$$

où $G_{\sigma}^{x}(y)=G_{\sigma}(x, y)$.

c) Pour $\delta(x, y)>r_{0}, \Delta_{\sigma}^{x} G_{\sigma}^{x}=0$. Si $\psi^{x}(y)=\mathrm{e}^{-\varphi_{x}(y)}, \Delta_{\sigma}^{x}\left(\frac{\psi^{x}}{G_{\sigma}^{x}}\right) \geq 0$ d'après ce qui a été dit précédemment.

d) De plus $\Delta_{\sigma}^{x}$ est sans terme constant et par hypothèse

$$
\lim _{y \rightarrow \infty} \frac{\psi^{x}(y)}{G_{\sigma}(y)} \leq C
$$

le principe du maximum appliqué à 1 et $\psi^{x} / G_{\sigma}^{x}$ pour $\Delta_{\sigma}^{x}$ dit que

$$
\psi^{x}(y) \leq C_{\sigma} G_{\sigma}^{x}(y)=C_{\sigma} G_{\sigma}(x, y)
$$

pour tout $y$ avec $\delta(x, y) \geq r_{0}$. Il existe $p_{0}, 1<p_{0} \leq 2$ tel que

$$
\left\|\int_{M} G_{\sigma}(x, y) \mathbf{1}_{\delta(x, y) \geq r_{0}}(y) f(y) \mathrm{d} \sigma(y)\right\|_{p_{0}} \leq C(p, \sigma)\|f\|_{p_{0}} .
$$

Soit $\mu^{2}=-\operatorname{Inf}\langle\Delta f, f\rangle /\left(f \in C_{0}^{\infty}(M),\|f\|_{2}\right)$ soit

$$
G_{\sigma}(x, y)=\int_{0}^{\infty} \mathrm{e}^{\sigma^{2} t} P_{t}(x, y) \mathrm{d} t
$$

où $P_{t}$ est la solution fondamentale de l'équation de la chaleur. On a déjà vu que $G_{\sigma}$ est fini partout, elle est même $C^{\infty}$ en dehors de la diagonale.

On considère l'ensemble $\Gamma=\{z, 0 \leq \operatorname{Re} z \leq 1\}$. On définit

$$
G_{z}(x, y)=\int_{0}^{\infty} \mathrm{e}^{\left(\mu^{2}-\varepsilon\right) z t} P_{t}(x, y) \mathrm{d} t
$$

Alors pour $\operatorname{Re} z=1,\left\|\int_{M} G_{z}(x, y) f(y) \mathrm{d} \sigma(y)\right\|_{2} \leq c\|f\|_{2}$. effet

Pour Re $z=0,\left\|\int_{M} G_{z}(x, y) f(y) \mathrm{d} \sigma(y)\right\|_{p} \leq c\|f\|_{p}$ pour tout $1<p \leq 2$. En

$$
\left|\int_{M} \int_{0}^{\infty} \mathrm{e}^{\left(u^{2}-\varepsilon\right) i t} P_{t}(x, y) \mathrm{d} t f(y) \mathrm{d} \sigma(y)\right| \leq \int_{M} \int_{0}^{\infty} P_{t}(x, y)|f(y)| \mathrm{d} \sigma(y) \mathrm{d} t .
$$

Comme $\mu^{2}>0$, on a $\left\|P_{t}\right\|_{2 \rightarrow 2} \leq \mathrm{e}^{-\mu^{2} t}$ pour $t>1$ et par interpolation

$$
\left\|P_{t}\right\|_{p \rightarrow p} \leq \mathrm{e}^{-2 \mu^{2} / p^{\prime} t} \quad \text { pour } t>1 \text {. }
$$

Il s'ensuit que

$$
\left\|G_{s} f\right\|_{p_{s}} \leq C(s)\|f\|_{p_{s}} \quad \text { où } \quad G_{s}=\int_{0}^{\infty} \mathrm{e}^{+\left(\mu^{2}-\varepsilon\right) s} P_{t}(x, y) \mathrm{d} t,
$$

$1 / p_{s}=\frac{1}{2}(1-s)+1 / p ;$ on peut choisir

$$
s=\frac{\sigma^{2}}{\mu^{2}-\varepsilon}, \quad \frac{1}{p_{s}}=\frac{1-\sigma^{2}}{\frac{1}{2}\left(\mu^{2}-2\right)}+\frac{\sigma^{2}}{\left(\mu^{2}-\varepsilon\right) p} .
$$


Comme $p$ est voisin de 1 et $\varepsilon$ arbitraire, on voit que pour tout $p$ tel que $p_{0}>\frac{1}{2}\left[1+\sigma^{2} / \mu^{2}\right]$, on a

$$
\left\|\int_{M} G_{\sigma}(x, y) f(y) \mathrm{d} \sigma(y)\right\|_{p_{0}} \leq C_{\sigma}\|f\|_{p_{0}} .
$$

Alors

$$
\begin{aligned}
& \left\|\int_{M} G_{\sigma}(x, y) \mathbf{1}_{\delta(x, y) \geq r_{0}}(y) f(y) \mathrm{d} \sigma(y)\right\|_{p_{0}} \leq C_{\sigma}\left(p_{0}\right)\|f\|_{p_{0}}, \\
& \left\|\int_{M} \psi_{\sigma}(x, y) \mathbf{1}_{\delta(x, y) \geq r_{0}}(y) f(y) \mathrm{d} \sigma(y)\right\|_{p_{0}} \leq C\left(\sigma, p_{0}\right)\|f\|_{p_{0}} .
\end{aligned}
$$

Par conséquent

$$
\left\|f_{2}\right\|_{p_{0}} \leq C\left(p, p_{0}\right)\|f\|_{p_{0}} \text { et }\left\|f^{*}\right\|_{p_{0}} \leq C\left(p_{0}\right)\|f\|_{p_{0}} .
$$

Comme l'inégalité est vraie pour $p=+\infty$, il s'ensuit que $\left\|f^{*}\right\|_{p} \leq C(p)\|f\|_{p}$ pour tout $p \geq p_{0}$.

Ce qui termine la preuve du théorème.

3.2. Démonstration du corollaire. - Nous n'insisterons pas sur la preuve de ce corollaire qui est une conséquence facile des théorèmes de comparaison voir [1].

\section{BIBLIOGRAPHIE}

[1] I. CHAVEL - Eigenvalues in Riemannian geometry, Pure and Applied Mathematics, vol. 115, Academic Press Inc., 1984, Including a chapter by Burton Randol, With an appendix by Jozef Dodziuk.

[2] J. L. ClerC \& E. M. STEIN - « $L^{p}$-multipliers for noncompact symmetric spaces », Proc. Nat. Acad. Sci. U.S.A. 71 (1974), p. 3911-3912.

[3] G. B. Folland \& E. M. Stein - « Estimates for the $\bar{\partial}_{b}$ complex and analysis on the Heisenberg group », Comm. Pure Appl. Math. 27 (1974), p. $429-522$.

[4] N. LohouÉ - «Fonction maximale de Hardy-Littlewood sur les variétés de Cartan-Hadamard», C. R. Acad. Sci. Paris 300 (1885).

[5] _ « Estimations $L^{p}$ des coefficients de représentation et opérateurs de convolution », Adv. in Math. 38 (1980), p. 178-221.

[6] _ _ « Sur les représentations uniformément bornées et le théorème de convolution de Kunze-Stein », Osaka J. Math. 18 (1981), p. 465-480.

TOME $135-2007-\mathrm{N}^{\mathrm{O}} 3$ 
[7] A. Nagel, E. M. Stein \& S. Wainger - «Balls and metrics defined by vector fields. I. Basic properties », Acta Math. 155 (1985), p. 103-147.

[8] A. Nevo - «Radial geometric analysis on groups », in Proceedings of first SAM Symposium on Discrete Geometric Analysis, Sendai 12-20 (Japan), 2002.

[9] N. T. Varopoulos, L. Saloff-Coste \& T. Coulhon - «Analysis and geometry on groups », Cambridge Tracts in Math. 100 (1992), p. 156. 